\title{
Interim Effectiveness and Safety Comparison of Bedaquiline-Containing Regimens for Treatment of Diabetic Versus Non-Diabetic MDR/XDR-TB Patients in China: A Multicenter Retrospective Cohort Study
}

Li Shi $\cdot$ Jingtao Gao $\cdot$ Mengqiu Gao $\cdot$ Ping Deng $\cdot$ Shu Chen $\cdot$ Minfu He $\cdot$ Wenjun Feng $\cdot$ Xiaoyun Yang Yunhui Huang $\cdot$ Fang He $\cdot$ Yumeng Hu $\cdot$ Liping Lei $\cdot$ Xuelian Li $\cdot$ Juan Du $\cdot$ Xiaomeng Hu $\cdot$ Zhi Liu $\cdot$ Peijun Tang · Junfeng Han · Hua Wang · Yi Han · Wei Shu $\cdot$ Yuxian Sun · Yi Pei · Yuhong Liu

Received: October 13, 2020 / Accepted: January 5, 2021 / Published online: January 30, 2021

(c) The Author(s) 2021

\section{ABSTRACT}

Introduction: Diabetes mellitus (DM), a common tuberculosis (TB) comorbidity, is associated with delayed bacillary clearance during anti-TB treatment and unfavorable outcomes. Bedaquiline (BDQ), when used as part of

Supplementary Information The online version contains supplementary material available at https:// doi.org/10.1007/s40121-021-00396-9.

Jingtao Gao and Mengqiu Gao are the co-first authors for this article.

L. Shi $\cdot$ P. Deng $\cdot$ W. Feng $\cdot$ X. Yang $\cdot$ Y. Huang F. He $\cdot$ Y. Hu $\cdot$ L. Lei · Y. Pei $(\bowtie)$

Department of Tuberculosis, Changsha Central Hospital, University of South China, Changsha 410007, China

e-mail: 1113284116@qq.com

J. Gao · W. Shu $\cdot$ Y. Sun · Y. Liu (ه)

Clinical Center on TB, Beijing Chest Hospital, Capital Medical University/Beijing Tuberculosis and Thoracic Tumor Research Institute, Beijing 101149, China

e-mail: liuyuhong0516@126.com

M. Gao · X. Li

Department of Tuberculosis, Beijing Chest Hospital, Capital Medical University/Beijing Tuberculosis and Thoracic Tumor Research Institute, Beijing 101149, China multidrug regimen for multidrug-resistant/extensively drug-resistant tuberculosis (MDR/ XDR-TB), has been shown to be effective and safe although treatment outcome and risks for patients with MDR/XDR-TB and DM are unknown. A multicenter retrospective study was conducted to compared the safety and effectiveness of 24-week BDQ-containing anti$\mathrm{TB}$ treatment for patients with MDR/XDR-TB with and without DM.

Methods: The study of patients with MDR/ XDR-TB with or without DM (enrolled February

\section{S. Chen · M. He}

Beijing Innovation Alliance of TB Diagnosis and Treatment, Beijing 101149, China

\section{J. Du $\cdot$ X. Hu}

Department of Tuberculosis, Wuhan Pulmonary Hospital, Wuhan 430030, China

\section{Z. Liu}

Department of Tuberculosis, Shenzhen Third People's Hospital, Shenzhen 518000, China

P. Tang

The Fifth People's Hospital of Suzhou, Suzhou 215000, China

J. Han

Tianjin Haihe Hospital, Tianjin 300000, China

H. Wang · Y. Han

Anhui Chest Hospital, Hefei 230000, China 
2018-September 2019, 21 Chinese hospitals) was supervised by the New Drug Introduction and Protection Program (NDIP). Of 640 patients with MDR/XDR-TB receiving BDQ-containing anti-TB treatments, two propensity score-matched groups (107 DM/107 non-DM) were compared for cumulative culture conversion rate, time to culture conversion, adverse events, and corrected QT interval.

Results: Body mass index was higher in patients with DM than patients without DM $(23.29 \pm 3.9$ vs. $20.5 \pm 3.6, \quad P<0.001)$; lung cavity prevalence $(86.9 \%$ vs. $72.9 \%, P=0.037)$ was also higher in patients with DM; the nonDM group had higher hepatitis prevalence $(29.0 \%$ vs. $15.9 \%, P=0.022)$. No significant intergroup differences were found for sputum culture conversion rate at week $8(80.0 \%$ vs. $81.4 \%, P=0.884)$, at week 24 (95.6\% vs. $98.2 \%$, $P=0.629)$, or for median time to sputum culture conversion [56 days (IQR 28-63) vs. 56 days $(I Q R ~ 28-84) \quad(P=0.687)]$. Favorable post-24week treatment outcomes were presented by $90.7 \%$ and $93.5 \%$ in the DM group and non-DM group, respectively, without significant intergroup differences $(P=0.448)$. The DM adverse event rate exceeded non-DM rate $(77.6 \%$ vs. $64.5 \%, P=0.035$ ).

Conclusion: Despite some differences in baseline characteristics, Chinese patients with MDR/ XDR-TB with or without DM had similar sputum culture conversion rates and favorable treatment outcomes post-24-week BDQ-containing anti-TB treatment. Low BMI but not DM is risk factor associated with unfavorable outcome of patients with MDR/XDR-TB.

Keywords: Adverse event; Bedaquiline; Diabetes; Multidrug resistant; Tuberculosis

\section{Key Summary Points}

Patients with MDR/XDR-TB and DM were more likely than patients without DM to exhibit clinically greater disease severity at baseline.
Patients with MDR/XDR-TB with and without DM undergoing treatment with BDQ-containing regimens achieved equally satisfactory sputum culture conversion rates.

All patients had acceptable safety and drug tolerability after 24 weeks of treatment.

\section{DIGITAL FEATURES}

This article is published with digital features, including a summary slide, to facilitate understanding of the article. To view digital features for this article go to https://doi.org/10.6084/ m9.figshare.13366676.

\section{INTRODUCTION}

In 2019, 463 million people aged 20-79 were afflicted with diabetes mellitus (DM) globally according to an International Diabetes Federation (IDF) report. This number is predicted to reach 578 million and 700 million by 2030 and 2045 [1], respectively. Meanwhile, the World Health Organization (WHO) recently reported an estimated 484,000 new cases of rifampicinresistant TB (RR-TB), of which 78\% were multidrug-resistant TB (MDR-TB) cases [2]. In China, which shoulders a double burden of DM and MDR-TB cases, numbers of patients with DM reached 116.4 million in 2019, ranking first in DM prevalence worldwide [1], while estimated MDR/RR-TB prevalence reached 66,000, ranking second worldwide [2]. Notably, DM is a major risk factor for progression of latent $\mathrm{TB}$ to active TB; DM triples future active TB risk and doubles future MDR-TB risk [3-5]. Several studies have shown that DM negatively impacts treatment outcomes in patients with MDR-TB. Specifically, DM was associated with both delayed Mycobacterium tuberculosis clearance during anti-tuberculosis (anti-TB) treatment and with worse anti-TB treatment outcomes that were, in turn, independently associated 
with increased risk of treatment failure and death [6-8]. Conversely, DM treatment outcomes can be affected by TB, since DM treatment success using hypoglycemic agents for controlling blood glucose levels can be undermined by TB itself and by certain anti-TB drugs [9]. Of additional concern is that increasing coprevalence of DM and TB may reverse decadeslong progress made against TB. The WHO now recommends careful DM screening prior to anti$\mathrm{TB}$ treatment initiation as interim policy [10].

Bedaquiline (BDQ), a newer agent for MDRTB treatment that was approved by the US Food and Drug Administration in 2012, is a diarylquinoline anti-mycobacterial drug that inhibits mycobacterial adenosine triphosphate (ATP) synthase [11]. Studies have shown that adding BDQ to a standard anti-MDR-TB treatment regimen reduced the time to sputum culture conversion, increased the proportion of patients with sustained negative sputum culture results, and improved patient long-term survival [11-13]. However, recently published clinical data related to the use of BDQ in patients with pulmonary MDR-TB and DM is limited.

A cohort of patients with MDR/XDR-TB treated with a BDQ-containing anti-TB regimen under China's New Drug Introduction and Protection Program (NDIP) was studied. The aim of the study was to assess safety and effectiveness of interim BDQ-containing anti-TB treatment in two groups of patients with MDR/ XDR-TB, those with and those without type 2 DM. Intergroup comparisons were conducted to provide evidence of safety and effectiveness of BDQ-containing anti-TB regimens when administered to patients with concurrent DM and MDR/XDR-TB.

\section{METHODS}

\section{Study Participants}

This retrospective study utilized an MDR/XDRTB cohort admitted between February 2018 and September 2019 that received follow-up until the end of March 2020 in 21 hospitals across China under a project named the New Drug
Introduction and Protection (NDIP) Program. With support from the China-Gates TB Control Project, NDIP was initiated in 2017 and established an effective nationwide working mechanism for the proper use of the first new anti-TB drug BDQ donated by the Global Drug Facility under an active drug safety monitoring framework. Medical professionals of selected TB specialized hospitals capable of MDR-TB diagnosis and treatment were well trained for patients enrollment, BDQ-containing regimen design and drug administration, treatment outcome and safety monitoring, and evaluation according to the NDIP protocol. A standardized electronic case report form was filled in by trained doctors in each center and data was reviewed by an independent data monitoring committee of NDIP routinely.

Patients were enrolled if they met the following eligibility. Inclusion criteria were (1) laboratory diagnosis of MDR/XDR-TB; (2) failure to respond to current MDR-TB regimens lacking bedaquiline; (3) at least 18 years of age; (4) no respiratory failure, cardiac failure, clinically significant arrhythmia, or corrected QT interval with Fridericia formula (QTcF) less than $450 \mathrm{~ms}$. Exclusion criteria were (1) allergy to BDQ; (2) participation in other clinical trials within the past 3 months; (3) pregnant or breast-feeding; (4) concomitant serious illness, including alanine aminotransferase/aspartate aminotransferase (ALT/AST) greater than three times the upper limits of normal (ULN) or total bilirubin greater than two times ULN, creatinine clearance less than $30 \mathrm{~mL} / \mathrm{min}$, hemoglobin less than or equal to $7.0 \mathrm{~g} / \mathrm{dL}$ and/or platelets less than $50 \times 10^{9} / \mathrm{L}$ at screening; (5) history of high-risk cardiac comorbidities (e.g., ventricular arrhythmia, myocardial infarction) with risk factors of QT prolongation: (a) electrocardiogram (ECG) at screening showing evident QT interval or $\mathrm{QTcF} \geq 450 \mathrm{~ms}$ (an unscheduled visit was allowed for ECG re-examination during the screening period to reevaluate patient eligibility); (b) pathologic Q wave ( $Q$ wave greater than $40 \mathrm{~ms}$ or depth of Q wave greater than $0.4-0.5 \mathrm{mV}$ ); (c) evidence of ventricular pre-excitation (e.g., Wolff-Parkinson-White syndrome); (d) ECG showed evidence of complete or clinically 
significant incomplete left bundle branch block or right bundle branch block; (e) evidence of grade II or III heart block; (f) intraventricular conduction delay, QRS duration greater than $120 \mathrm{~ms}$; (g) bradycardia (sinus heart rate less than $50 \mathrm{bpm}$ ); (h) personal or family history of long QT syndrome; (i) history of heart disease, symptomatic or asymptomatic arrhythmia (except for sinus arrhythmia); (j) cardiogenic syncope; or (k) have risk factors for developing torsades de pointes (TdP), such as heart failure, hypokalemia, or hypomagnesemia. NDIP was approved by the ethics committee of each participating hospital. All patients enrolled provided written informed consent.

\section{Treatment Regimen}

According to WHO guidelines and NDIP protocol, local physicians developed individualized background regimens based on patients' previous histories of anti-TB treatment and drug susceptibility testing (DST) results as well as drug tolerance. For patients with DST results, BDQ was used in combination with at least three background drugs to which their TB isolate was susceptible. For patients without definitive DST results, BDQ was used in combination with at least four drugs to which the isolate was likely to be susceptible on the basis of treatment history and local epidemiology of drug resistance. BDQ was administered at the recommended dose of $400 \mathrm{mg}$ once a day for 14 days and then at a dose of $200 \mathrm{mg}$ three times per week for the remaining 22 weeks.

Background regimens consisted of the antiTB drug formulations guided by DST, including moxifloxacin, levofloxacin, linezolid, clofazimine, amikacin, capreomycin, protionamide, cycloserine, pyrazinamide, ethambutol, paraaminosalicylic acid, high-dose isoniazid, meropenem, and amoxicillin/clavulanate. To ensure patient adherence to outpatient treatment, patients were supervised by trained professional clinicians, who monitored patient treatment progress and provided medical and psychosocial support.

Prolongations of QTcF more than $500 \mathrm{~ms}$ were investigated, and treatment was modified if the prolongation was considered to be drug related by the site investigators.

\section{Treatment Evaluation}

Patients were followed every 2 weeks for the first month and every 4 weeks thereafter. Information regarding demographic characteristics, clinical history, medication history, laboratory test results, electrocardiographic (ECG) and adverse events (AEs), and bacteriological and radiological findings were collected from the NDIP information monitoring system. Monitored patient outcomes included cumulative sputum culture conversion rate, time to sputum culture conversion, adverse events (AEs), and QTcF. Culture conversion was defined as presence of a positive sputum culture result at baseline followed by at least two consecutive negative MGIT cultures of sputum taken at least 28 days apart. Time to culture conversion was defined as the time from baseline to the first negative sputum culture in patients exhibiting culture conversion. AEs were recorded during the treatment period and graded according to the Division of AIDS Table for Grading the Severity of Adult and Pediatric Adverse Events guidelines, version 2.1 [14].

Patient outcomes at week 24 were graded as favorable if the patient had completed 24 weeks of BDQ treatment and achieved sustained sputum culture conversion without interruption of treatment, or if the patient had consistently tested sputum culture negative relative to baseline. Those who died during treatment, failed to achieve culture conversion or reversion, prematurely discontinued the study, or were otherwise lost to follow-up were deemed to have unfavorable outcomes.

\section{Statistical Analysis}

Qualitative and quantitative variables were reported in percentages with mean \pm standard deviation (SD) and median interquartile range (IQR) values, as appropriate. Clinical characteristics of patients with MDR-TB with and without DM were compared between groups using chi-square test or Fisher's exact test for 
categorical variables and Student's $t$ test or Mann-Whitney $U$ test for continuous variables. To elucidate potential factors associated with unfavorable interim outcomes, selected variables with $P$ values less than 0.05 as determined via univariate regression analysis were further analyzed using multivariate logistic regression with the "backward selection" method. Cox hazard proportional models were used to determine time to culture conversion results.

Patients with DM (the DM group) and patients without DM (the non-DM group) were matched in a 1:1 ratio on the basis of age, sex, and diagnosis with DST profiles using propensity score matching (PSM). Effectiveness and safety were evaluated for the matched cohort, with PSM conducted using the $\mathrm{R}$ software MatchIt package. Data analyses were performed using SPSS 22.0 (IBM, Armonk, NY, USA). $P$ values less than 0.05 were considered statistically significant.

\section{Compliance with Ethics Guidelines}

The study was conducted in accordance with the 1964 Helsinki declaration and its later amendments or comparable ethical standards. This study protocol was approved by the ethics committee of each participating hospital. Beijing Chest Hospital is the leader of the study with reference number of (No. 5 of 2018). The study was also approved by the ethics committees of the following hospitals: Changsha Central Hospital affiliated to University of South China, Chest Hospital of Jiangxi Province, Anhui Chest Hospital, Tianjin Haihe Hospital, Wuhan Pulmonary Hospital, The Fifth People's Hospital of Suzhou, The Third People's Hospital of Shenzhen, Guiyang Public Health Center, Fuzhou Pulmonary Hospital, Guangzhou Chest Hospital, Wenzhou Central Hospital, Heilongjiang Tuberculosis Prevention and Control Institute, Qingdao Chest Hospital, Jilin Tuberculosis Prevention and Control Institute, Hangzhou Red Cross Hospital, Shenyang Chest Hospital, Hainan Second People's Hospital, Nanjing Second People's Hospital, The Sixth People's Hospital of Zhengzhou and Hebei Chest Hospital. Each patient provided their informed consent before they were enrolled into NDIP.

\section{RESULTS}

\section{Baseline Characteristics of Patients with MDR/XDR-TB with and Without DM}

Of 640 patients with MDR/XDR-TB treated with BDQ-containing regimens from 21 centers of NDIP, 107 (16.7\%) had type 2 DM. When PSM was used at $1: 1$, another 107 patients with MDR/XDR-TB without DM were matched on the basis of age, gender, and DST profiles at baseline (Table 1). Body mass index (BMI) values of patients with MDR/XDR-TB in the DM group exceeded those of the non-DM group $(23.29 \pm 3.9$ vs. $20.5 \pm 3.6, \quad P<0.001)$. Comorbid viral hepatitis infection was more common in the non-DM group $(29.0 \%$ vs. $15.9 \%, P=0.022)$. Patients with DM were more likely to have lung cavities $(86.9 \%$ vs. $72.9 \%$, $P=0.010)$. No statistically significant intergroup differences in other clinical characteristics at baseline (e.g., previous duration of TB illness, extrapulmonary $\mathrm{TB}$, and blood albumin level, etc.) were observed $(P>0.05)$.

Regarding patients with DM, median lifetime duration with DM was 72 months (IQR 20-120), median fasting plasma glucose was $7.7 \mathrm{mmol} / \mathrm{L}$ (IQR 5.7-9.5), median glycosylated hemoglobin level was 7.3 (IQR 6.2-8.7), and numbers of patients with DM using insulin, insulin plus an oral medicine, oral medicine, and diet control as DM treatments were 56 (52.3\%), 31 (29.0\%), $13(12.2 \%)$, and $7(6.5 \%)$, respectively.

\section{Optimal Background Anti-TB Drug Regimen}

As per the protocol recommended by NDIP, a median of 5 (IQR 4-5) anti-TB drugs were used to formulate the optimal background regimen, including fluoroquinolones, linezolid, clofazimine, and cycloserine as major components. Other drugs and proportions of patients receiving them are presented in Table 2 , with no differences observed between groups. 
Table 1 Clinical characteristics of patients with MDR/XDR-TB with or without DM at the baseline

\begin{tabular}{|c|c|c|c|}
\hline Characteristics & $\begin{array}{l}\text { DM group } \\
(n=107)\end{array}$ & $\begin{array}{l}\text { Non-DM group } \\
(n=107)\end{array}$ & $P$ value \\
\hline Mean age, years (SD) & $49.8 \pm 10.5$ & $49.3 \pm 9.7$ & 0.736 \\
\hline Male, no. (\%) & $98(91.6)$ & $98(91.6)$ & $>0.999$ \\
\hline Mean BMI, kg/m² (SD) & $23.29 \pm 3.9$ & $20.5 \pm 3.6$ & $<0.001$ \\
\hline Patients with previous anti-TB treatment history, no. (\%) & $90(84.1)$ & $96(89.7)$ & 0.224 \\
\hline DST profile, no. (\%) & & & $>0.999$ \\
\hline MDR-TB & $36(33.6)$ & $36(33.6)$ & \\
\hline Pre-XDR-TB & $39(36.5)$ & $39(36.5)$ & \\
\hline XDR-TB & $32(29.9)$ & $32(29.9)$ & \\
\hline Median duration of TB, months (IQR) & $30.0(9.0-72.0)$ & $44.0(12.0-110.0)$ & 0.170 \\
\hline Site of TB focus, no. (\%) & & & 0.252 \\
\hline Pulmonary only & $103(96.3)$ & $98(91.6)$ & \\
\hline Pulmonary and extrapulmonary & $4(3.7)$ & $9(8.4)$ & \\
\hline Lung cavities, no. (\%) & & & 0.010 \\
\hline Without any cavities & $14(13.1)$ & $29(27.1)$ & \\
\hline With at least 1 cavity & $93(86.9)$ & $78(72.9)$ & \\
\hline \multicolumn{4}{|l|}{ Comorbidities, no. (\%) } \\
\hline Heart disease & $4(3.7)$ & $3(2.8)$ & 0.701 \\
\hline Hepatitis infection & $17(15.9)$ & $31(29.0)$ & 0.022 \\
\hline Median duration of DM, months (IQR) & $72(20-120)$ & - & $\mathrm{N} / \mathrm{A}$ \\
\hline DM medications, no. (\%) & & - & $\mathrm{N} / \mathrm{A}$ \\
\hline Insulin & $56(52.3)$ & & \\
\hline Oral hypoglycemic ${ }^{\mathrm{a}}$ & $31(29.0)$ & & \\
\hline Both & $13(12.2)$ & & \\
\hline None & $7(6.5)$ & & \\
\hline Median fasting plasma glucose, $\mathrm{mmol} / \mathrm{L}$ (IQR) & $7.7(5.7-9.5)$ & - & $\mathrm{N} / \mathrm{A}$ \\
\hline Median glycosylated hemoglobin (IQR) & $7.3(6.2-8.7)$ & - & $\mathrm{N} / \mathrm{A}$ \\
\hline Mean blood albumin, g/L (SD) & $39.7 \pm 5.9$ & $38.7 \pm 5.0$ & 0.164 \\
\hline
\end{tabular}

$B M I$ body mass index, $S D$ standard deviation, $I Q R$ interquartile range, $D S T$ drug susceptibility testing, $M D R-T B$ multidrugresistant tuberculosis, Pre-XDR-TB tuberculosis resistant to either a fluoroquinolone or a second-line injectable drug, $X D R-$ $T B$ extensively drug-resistant tuberculosis, $N / A$ not applicable

a Included metformin, acarbose, and thiazolidinediones 
Table 2 Optimal background anti-TB drugs used in patients with MDR/XDR-TB with or without DM

\begin{tabular}{|c|c|c|c|}
\hline Drugs, no. (\%) & $\begin{array}{l}\text { DM } \\
\text { group } \\
(n=107)\end{array}$ & $\begin{array}{l}\text { Non-DM } \\
\text { group } \\
(n=107)\end{array}$ & $P$ value \\
\hline Moxifloxacin & $51(47.7)$ & $59(55.1)$ & 0.274 \\
\hline Levofloxacin & $10(9.3)$ & $11(10.3)$ & 0.818 \\
\hline Linezolid & $97(90.7)$ & $96(89.7)$ & 0.818 \\
\hline Clofazimine & $77(72.0)$ & $69(64.5)$ & 0.240 \\
\hline Cycloserine & $91(85.0)$ & $89(83.2)$ & 0.708 \\
\hline Ethambutol & $12(11.2)$ & $9(8.4)$ & 0.491 \\
\hline Pyrazinamide & $24(22.4)$ & $19(17.8)$ & 0.394 \\
\hline Protionamide & $49(45.8)$ & $55(51.4)$ & 0.412 \\
\hline Amikacin & $31(29.0)$ & $42(39.3)$ & 0.113 \\
\hline Capreomycin & $22(20.6)$ & $25(23.4)$ & 0.620 \\
\hline p-Aminosalicylic acid & $43(40.2)$ & $33(30.8)$ & 0.153 \\
\hline Pasiniazid & $2(1.9)$ & $2(1.9)$ & $>0.999$ \\
\hline $\begin{array}{l}\text { Amoxicillin/clavulanic } \\
\text { acid }\end{array}$ & $12(11.2)$ & $11(10.3)$ & 0.825 \\
\hline Streptomycin & $1(0.9)$ & $0(0)$ & $>0.999$ \\
\hline Clarithromycin & $3(2.8)$ & $0(0)$ & 0.246 \\
\hline High-dose isoniazid & $2(1.9)$ & $1(0.93)$ & $>0.999$ \\
\hline $\begin{array}{l}\text { Median no. of drugs in } \\
\text { background regimen, } \\
\text { (IQR) }\end{array}$ & $5(4-5)$ & $5(4-5)$ & 0.364 \\
\hline
\end{tabular}

Table 3 Sputum culture conversion in two groups

\begin{tabular}{cccc}
\hline Sputum conversion & $\begin{array}{l}\text { DM } \\
\text { group }\end{array}$ & $\begin{array}{l}\text { Non-DM } \\
\text { group }\end{array}$ & $\boldsymbol{P}$ value \\
\hline Culture conversion at & $60 / 75$ & $48 / 59$ & 0.844 \\
8th week, $n / N(\%)$ & $(80.0)$ & $(81.4)$ & \\
Culture conversion at & $66 / 69$ & $54 / 55$ & $0.629^{\mathrm{a}}$ \\
24th week, $n / N(\%)$ & $(95.6)$ & $(98.2)$ & \\
Time to culture & 56.0 & 56.0 & 0.876 \\
conversion, days & $(28-63)$ & $(28-84)$ & \\
\hline
\end{tabular}

a Fisher's exact test is used

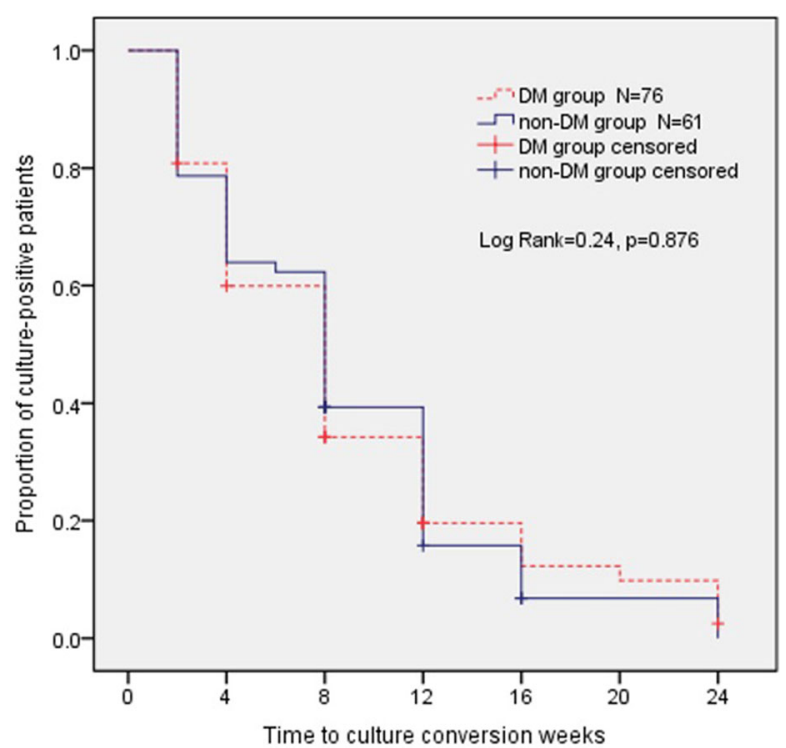

Fig. 1 Kaplan-Meier plot for time to sputum culture conversion within 24 weeks

\section{Interim Sputum Culture Conversion and Treatment Outcomes}

Comparisons of sputum culture conversion rates and time to sputum culture conversion between groups are outlined in Table 3 . Among 107 patients with MDR/XDR-TB in the DM group, $76(71 \%)$ were culture positive at baseline, of which $60(80.0 \%)$ achieved early culture conversion after 8 weeks and 66 (95.6\%) completed 24 weeks of intensive treatment with sustained culture conversion. The median time to sputum culture conversion was 56 days (IQR 28-63).

Among the matched 107 patients with MDR/ XDR-TB in the non-DM group, 61 patients $(57 \%)$ were culture positive at baseline, of which 48 (81.4\%) achieved early culture conversion after 8 weeks and 54 (98.2\%) eventually completed 24 weeks of intensive treatment with sustained culture conversion. The median time to conversion was also 56 days (IQR 28-84).

According to time to event analysis, no significant intergroup differences in probability of achieving culture conversion were observed by the end of the 24-week treatment period $(P=0.876$; Fig. 1$)$. 
Table 4 Interim effectiveness of treatment in patients with MDR/XDR-TB at 24th week in two groups

\begin{tabular}{|c|c|c|c|}
\hline $\begin{array}{l}\text { Interim treatment } \\
\text { outcome, no. (\%) }\end{array}$ & $\begin{array}{l}\text { DM group } \\
(n=107)\end{array}$ & $\begin{array}{l}\text { Non-DM } \\
\text { group } \\
(n=107)\end{array}$ & $P$ value \\
\hline Favorable outcome & $97(90.7)$ & $100(93.5)$ & 0.448 \\
\hline $\begin{array}{l}\text { Sputum culture } \\
\text { conversion }\end{array}$ & $66(61.7)$ & $54(50.5)$ & \\
\hline Completion & $31(29.0)$ & $46(43.0)$ & \\
\hline $\begin{array}{l}\text { Unfavorable } \\
\text { outcome }\end{array}$ & $10(9.3)$ & $7(6.5)$ & 0.448 \\
\hline Death & $0(0.0)$ & $0(0.0)$ & \\
\hline Transfer out & $4(3.7)$ & $4(3.7)$ & \\
\hline Default & $3(2.8)$ & $2(1.9)$ & \\
\hline $\begin{array}{l}\text { Sputum culture } \\
\text { reversion }\end{array}$ & $3(2.8)$ & $1(0.9)$ & \\
\hline
\end{tabular}

On the basis of interim treatment outcomes at 24 weeks post-treatment initiation for the 107 patients with MDR/XDR-TB in each group outlined in Table 4, including 97 (90.7\%) in the DM group and $100(93.5 \%)$ in the non-DM group, favorable outcomes with no significant intergroup differences were observed $(P=0.448)$. Meanwhile, $4 \quad(3.7 \%)$ patients transferred out, 3 (2.8\%) patients defaulted, and $3(2.8 \%)$ patients experienced sputum culture reversion within 24 weeks in the DM group, with similar distributions observed for the nonDM group and no significant intergroup differences observed $(P=0.448)$.

Multivariable logistic regression analysis showed that DM comorbidity was not associated with unfavorable treatment outcomes at the end of 24 weeks [odds ratio (OR) 1.169, 95\% confidence interval (CI) 0.417-3.276, $P=0.767$ ], while BMI less than $18.5 \mathrm{~kg} / \mathrm{m}^{2}$ (OR $2.921,95 \%$ CI $1.030-8.280, P=0.044$ ) was a risk factor associated with unfavorable outcomes (Table S1).
Table 5 Frequency of adverse events reported in two groups

\begin{tabular}{|c|c|c|c|}
\hline $\begin{array}{l}\text { Adverse event, } \\
\text { no. (\%) }\end{array}$ & $\begin{array}{l}\text { DM } \\
\text { group } \\
(n=107)\end{array}$ & $\begin{array}{l}\text { Non-DM } \\
\text { group } \\
(n=107)\end{array}$ & $P$ value \\
\hline $\begin{array}{l}\text { Electrocardiogram } \\
\text { abnormality }\end{array}$ & $48(44.9)$ & $49(45.8)$ & 0.891 \\
\hline $\begin{array}{l}\text { QTcF } \\
\text { abnormalities }\end{array}$ & $42(39.3)$ & $43(40.2)$ & \\
\hline Others ${ }^{a}$ & $6(5.6)$ & $6(5.6)$ & \\
\hline Hepatotoxicity & $41(38.3)$ & $18(16.8)$ & $<0.001$ \\
\hline Renal injury & $13(12.1)$ & $8(7.5)$ & 0.251 \\
\hline Optic neuritis & $7(6.5)$ & $6(5.6)$ & 0.775 \\
\hline Ototoxicity & $4(3.7)$ & $6(5.6)$ & 0.746 \\
\hline $\begin{array}{l}\text { Hematological } \\
\text { injury }\end{array}$ & $12(11.2)$ & $12(11.2)$ & $>0.999$ \\
\hline $\begin{array}{l}\text { Gastrointestinal } \\
\text { symptoms } \\
\text { response }\end{array}$ & $11(10.3)$ & $4(3.7)$ & 0.108 \\
\hline $\begin{array}{l}\text { Peripheral } \\
\text { neuropathy }\end{array}$ & $4(3.7)$ & $14(13.1)$ & 0.027 \\
\hline $\begin{array}{l}\text { Dermatologic } \\
\text { reaction }\end{array}$ & $0(0)$ & $2(1.9)$ & 0.498 \\
\hline Arthralgia & $3(2.8)$ & $0(0)$ & 0.246 \\
\hline Hemoptysis & $5(4.7)$ & $2(1.9)$ & 0.442 \\
\hline $\begin{array}{l}\text { Psychiatric } \\
\text { symptoms }\end{array}$ & $6(5.6)$ & $3(2.8)$ & 0.496 \\
\hline $\begin{array}{l}\text { Electrolyte } \\
\text { disturbance }\end{array}$ & $4(3.7)$ & $1(0.9)$ & 0.369 \\
\hline $\begin{array}{l}\text { Headache and } \\
\text { dizziness }\end{array}$ & $6(5.6)$ & $0(0)$ & 0.029 \\
\hline Fever & $2(1.9)$ & $0(0)$ & 0.498 \\
\hline Others ${ }^{\mathrm{b}}$ & $4(3.7)$ & $1(0.9)$ & 0.369 \\
\hline
\end{tabular}


Table 6 QTcF profiles in two groups

\begin{tabular}{llll}
\hline QTcF, ms & $\begin{array}{l}\text { DM group } \\
(\boldsymbol{n}=\mathbf{1 0 7})\end{array}$ & $\begin{array}{l}\text { Non-DM } \\
\text { group } \\
(\boldsymbol{n}=\mathbf{1 0 7})\end{array}$ & P value \\
\hline $\begin{array}{llll}\text { Mean baseline } \\
\text { QTcF (SD) }\end{array}$ & $412.1 \pm 22.4$ & $404.6 \pm 24.7$ & 0.021 \\
Mean QTcF & $424.9 \pm 28.3$ & $420.7 \pm 28.6$ & 0.005 \\
during & & & \\
treatment & & & \\
(SD) & & & \\
Patients with at & $42(39.3)$ & $43(40.2)$ & 0.889 \\
least one & & & \\
QTcF greater & & & \\
than 450 ms, & & & \\
no. (\%) & & & \\
Patients with at & $15(14.0)$ & $20(18.7)$ & \\
least one & & & \\
QTcF & & & \\
increment & & & \\
from baseline & & & \\
greater than & & & \\
60 ms, no. & & & \\
(\%) & & & \\
Patients with at & $4(3.7)$ & & \\
least one & & & \\
QTcF greater & & & \\
than 500 ms, & & & \\
no. (\%) & & & \\
\hline
\end{tabular}

QTcF QT interval with Fridericia correction

\section{Safety}

In the DM group, 83 patients $(77.6 \%)$ reported a total of 170 AEs within the 24-week intensive treatment period. The most commonly reported AEs were ECG abnormalities (44.9\%), hepatotoxicity (i.e., liver enzyme abnormalities) $(38.3 \%)$, renal injury $(12.1 \%)$, hematological injury $(11.2 \%)$, and gastrointestinal symptoms response $(10.3 \%)$.

In the non-DM group, 69 patients $(64.5 \%)$ reported a total of 126 AEs during the 24-week intensive treatment course. The most commonly reported AEs in this group were ECG abnormalities (45.8\%), hepatotoxicity (16.8\%), peripheral neuropathy (13.1\%), hematological injury (11.2\%), and renal injury (7.5\%). Other AEs and proportions of patients experiencing them are shown in Table 5. Except for hepatotoxicity, peripheral neuropathy, and headache and dizziness, no significant intergroup differences in adverse event rates were reported and no deaths occurred.

Table 6 presents QTcF profiles of the two groups. The mean baseline QTcF of patients with MDR/XDR-TB and DM was $412.1 \mathrm{~ms}$ $(\mathrm{SD}=22.4)$, which is higher than that of patients without DM $(P=0.021)$. The mean QTcF of both groups increased during the 24-week treatment with the aforementioned BDQ-containing anti-TB regimen, with significant intergroup differences observed $(P=0.005)$.

Numbers of patients with DM and at least one $\mathrm{QTCF} \geq 500 \mathrm{~ms}$ or one increment of $\mathrm{QTcF} \geq 60 \mathrm{~ms}$ from baseline were $4(3.7 \%)$ and 15 (14\%), respectively, with corresponding numbers of patients without DM of $4(3.7 \%)$ and $20(18.7 \%)$ observed and no significant intergroup differences observed (DM: $P>0.999$; non-DM: $P=0.355)$. For the eight patients whose QTcF values reached the critical threshold of $500 \mathrm{~ms}$, ECG and electrolyte measurements were repeated and four patients with DM continued BDQ administration with normal electrolyte levels and QTcF values (less than $500 \mathrm{~ms}$ ) were observed when repeated; meanwhile, one in four of these patients without DM permanently discontinued $\mathrm{BDQ}$, due to persistently high QTcF levels noted after repeated ECG monitoring and confirmation.

\section{DISCUSSION}

Findings of previous studies suggested that DM may undermine the absorption, distribution, biotransformation, and excretion of many pharmaceutical agents [15]. Given bedaquiline's unique pharmacokinetic profile [16], its pharmacokinetics in patients with DM may be altered by changes in subcutaneous adipose 
blood flow, gastric emptying, or nephropathy, and also by possible drug-drug interactions with hypoglycemic agents [17]. Although BDQ has been used to treat MDR/XDR-TB for years, few studies related to efficacy and safety of BDQ-containing anti-TB regimens administered to patients with DM have been reported, prompting us to conduct this first study to date to investigate this topic.

In this respective cohort study, $16.7 \%$ of patients with MDR/XDR-TB had DM, mirroring DM/DR-TB rates reported in South Korea [6]. In the African region, the most common MDR/ XDR-TB comorbidity is HIV, while in the Western Pacific region, DM is a more common comorbidity in this patient group. Such findings have influenced policy making and implementation measures by regional or national TB Programs (NTP). In order to maintain consistency of key intergroup baseline characteristics, here a control without DM was matched with a comparable patient with DM for each social demographic factor (age and sex) and DST profile in a 1:1 ratio between groups. Mirroring observations reported in other studies [18], patients with MDR/XDR-TB and DM were more likely to exhibit baseline clinical signs associated with greater disease severity (e.g., positive bacteriological status, lung cavities). Here we also found that patients with MDR/XDR-TB with lower BMIs (less than $18.5 \mathrm{~kg} / \mathrm{m}^{2}$ ) tended to have unfavorable treatment outcomes (OR $2.291,95 \%$ CI 1.030-8.280, $P=0.044$ ), mirroring findings reported by our team earlier this year [19]. Meanwhile, no significant statistical intergroup differences were observed for baseline characteristics of other anti-TB drugs used to formulate optimal background regimens. Although baseline DM group disease severity exceeded that of the group of patients without DM, no significant intergroup differences were observed in median time to culture conversion (56 days, IQR 28-63 days vs. 56 days, IQR 28-84 days, $P=0.876$ ) or culture conversion rates (80.0\% vs. $81.4 \%, P=0.844)$ at 8 weeks and (95.6\% vs. $98.2 \%, P=0.629)$ at 24 weeks of antiTB treatment. Notably, the aforementioned median time to culture conversion results found here mirrored results reported by Salhotra et al. [20] and Borisov et al. [21], while the 24-week culture conversion rate found here exceeded the 6-month culture conversion rate reported in a South Korean study $(80 \%, n=21)$ [22] and the rate of $78 \%(n=537)$ reported in a multinational study (France, Georgia, Armenia, South Africa) [23].

It is widely accepted that metformin and insulin analogues used to control high blood glucose levels also possess anti-inflammatory properties that render these hypoglycemic agents useful for treating severe infections, such as MDR/XDR-TB with comorbid DM [17]. Indeed, the inclusion of insulin and metformin or both agents in treatment regimens for the vast majority of patients with DM has likely already benefited patients afflicted with both MDR/XDR-TB and DM. Nevertheless, findings of this study suggest that even in patients with MDR/XDR-TB and DM who receive hypoglycemic agent-based treatments, favorable treatment outcomes (e.g., rapid time to culture conversion results, optimal culture conversion rates) are still attainable with 24-week-long intensive anti-TB treatment with BDQ-containing regimens.

Many AEs experienced during MDR/XDR-TB treatment have been attributed to comorbid DM [24]. Since BDQ increased all-cause mortality rates of patients with MDR/XDR-TB in a phase IIb trial, BDQ toxicity has always been a major concern, especially for patients with MDR/XDR-TB and DM. Here we also found that patients with MDR/XDR-TB and DM reported AEs more frequently than did their non-DM counterparts, with most frequently observed AEs in both groups of ECG abnormalities and hepatotoxicity. With regard to ECG abnormalities, mean baseline QTcF and mean QTcF values of MDR/XDR-TB DM group patients during the 24-week treatment course were higher than respective non-DM group values, although no significant differences from baseline between groups were noted in occurrence of $\mathrm{QTcF} \geq 500 \mathrm{~ms}$ or increment of QTcF $\geq 60 \mathrm{~ms}$.

BDQ is metabolized primarily through hepatic metabolic pathways that are shared with several oral hypoglycemic agents (e.g., thiazolidinedione, acarbose); thus, hepatic-related adverse reactions may develop more frequently when BDQ and hypoglycemic agents are used 
together [17]. Consequently, here $38.3 \%$ of patients with MDR/XDR-TB and DM reported incidents of hepatotoxicity manifesting primarily as liver enzyme (AST/ALT) elevations, significantly more than the non-DM group rate (16.8\%) $(P<0.001)$. Even though occurrences of AEs, such as peripheral neuropathy, headache, and dizziness, were more commonly reported by patients with DM, most of these AEs were of grade 1 or 2 in severity, with few grade 3 or 4 AEs and no grade 5 AEs observed in either group as evidence for safety and tolerability of BDQ-containing regimens when administered to both groups.

In 2015, the WHO released its active TB drug safety monitoring and management (aDSM) framework, which described how to implement active pharmacovigilance in order to quickly detect, manage, and report suspected or confirmed drug toxicities within the context of new drugs and short treatment regimens. The first global report for surveillance of adverse event of 26 countries recently demonstrated that aDSM was feasible and the overall proportion of AEs was reasonably low $(8.7 \%$ of patients with serious AEs (grade 3 and 4, no grade 5 AEs) with treatment regimens including $\mathrm{BDQ}$ and delamanid (DLM) [25]. aDSM was implemented since NDIP initiated in China and provided valuable information on the AEs in Chinese patients treated with new and repurposed antiTB drugs, including the subpopulations with DM in this study.

Recently, the role of BDQ and/or DLM in patients with DR-TB and comorbidities such as human immunodeficiency virus (HIV), hepatitis $\mathrm{C}$ virus (HCV) coinfection, or DM have been reported. Olayanju et al. found that there were no significant differences in 6-month culture conversion and 18-month favorable outcome rate in the BDQ versus BDQ-DLM combination group in HIV-infected patients. Furthermore, the BDQ-DLM combination regimen presented comparable long-term safety to BDQ-based regimen in patients with DR-TB irrespective of HIV status [26]. Franke et al., on behalf of the endTB observational study team, reported culture conversion of patients with MDR-TB with BDQ and/or DLM, from 17 epidemiologically diverse countries with comorbidities such as
HIV, HCV, or diabetes. Patients with HIV had lower probability of conversion than patients without HIV. Patients with HCV or DM/glucose intolerance were not associated with culture conversion [27].

This study had several limitations. First, as inherent to all retrospective studies, DM diagnosis was based on medical records since we were unable to track patient fasting glucose levels and glycosylated hemoglobin data monthly to assess effects of glycemic control measures on treatment outcomes. Second, we assessed initial TB severity based on pulmonary imaging findings for patients in both groups at baseline but were unable to assess pulmonary cavity closure at 24 weeks, hindering our evaluation of treatment results. Third, we focused on interim outcomes at 24 weeks rather than final outcomes based on sputum culture. Further studies are warranted to determine final treatment outcomes and relapse rates in this cohort. Finally, despite few reported culture reversion, short-term follow-up may lead to overestimation of culture conversion rates.

\section{CONCLUSION}

This study demonstrated that patients with MDR/XDR-TB and DM were more likely than patients without DM to exhibit clinically greater disease severity at baseline. Nevertheless, patients with MDR/XDR-TB with and without DM undergoing treatment with $\mathrm{BDQ}$ containing regimens achieved equally satisfactory sputum culture conversion rates with acceptable safety and drug tolerability observed for all patients after 24 weeks of treatment.

\section{ACKNOWLEDGEMENTS}

We expressed our thanks to Long Jin, Ming Wang, Pengfei Ren, Qi Qi, Chunmei Hu, Qiuping $\mathrm{Wu}, \mathrm{Mei}$ Lin, Huailong Jiang, Na Yang, Saiduo Liu, Dan Yi, Dan Cui, Yuqing $\mathrm{Wu}$, and Linchun $\mathrm{Wu}$ for their time and efforts in patients' follow-up and data collection. We also acknowledge the outstanding contributions 
from the Clinical Center on Tuberculosis, China CDC and all workers of the NDIP program.

Funding. This work was supported by Science and Technology Bureau Project of Changsha (grant number 2018-kq1801145); Hunan Administration of Traditional Chinese Medicine (grant number 201890) and Scientific Research Project of Hunan Provincial Health Commission (grant number 20201439), the journal's Rapid Service was supported by NDIP project.

Authorship. All named authors meet the International Committee of Medical Journal Editors (ICMJE) criteria for authorship for this article, take responsibility for the integrity of the work as a whole, and have given their approval for this version to be published.

Authors' Contributions. LS, JG, MG, YL, PD, SC, MH, and YP contributed to the study conception and design. Patient management and data collection were performed by $\mathrm{WF}, \mathrm{XY}, \mathrm{YH}$, $\mathrm{FH}, \mathrm{YH}, \mathrm{LL}, \mathrm{XL}, \mathrm{XH}, \mathrm{ZL}, \mathrm{PT}, \mathrm{JH}, \mathrm{HW}, \mathrm{JD}$, and $\mathrm{YH}$, and statistical analysis was performed by LS, WS, and YS. The first draft of the manuscript was written by LS and JG. MG, YL, and YP commented on previous versions of the manuscript. All authors read and approved the final manuscript. LS, JG, and MG are the co-first authors and contributed equally to this study.

Disclosures. Li Shi, Jingtao Gao, Mengqiu Gao, Ping Deng, Shu Chen, Minfu He, Wenjun Feng, Xiaoyun Yang, Yunhui Huang, Fang He, Yumeng $\mathrm{Hu}$, Liping Lei, Xuelian Li, Juan Du, Xiaomeng $\mathrm{Hu}$, Zhi Liu, Peijun Tang, Junfeng Han, Hua Wang, Yi Han, Wei Shu, Yuxian Sun, Yi Pei, and Yuhong Liu have nothing to disclose.

Compliance with Ethics Guidelines. The study was conducted in accordance with the 1964 Helsinki declaration and its later amendments or comparable ethical standards. This study protocol was approved by the ethics committee of each participating hospital. Beijing Chest Hospital is the leader of the study with reference number of (No. 5 of 2018). The study was also approved by the ethics committees of the following hospitals: Changsha Central Hospital affiliated to University of South China, Chest Hospital of Jiangxi Province, Anhui Chest Hospital, Tianjin Haihe Hospital, Wuhan Pulmonary Hospital, The Fifth People's Hospital of Suzhou, The Third People's Hospital of Shenzhen, Guiyang Public Health Center, Fuzhou Pulmonary Hospital, Guangzhou Chest Hospital, Wenzhou Central Hospital, Heilongjiang Tuberculosis Prevention and Control Institute, Qingdao Chest Hospital, Jilin Tuberculosis Prevention and Control Institute, Hangzhou Red Cross Hospital, Shenyang Chest Hospital, Hainan Second People's Hospital, Nanjing Second People's Hospital, The Sixth People's Hospital of Zhengzhou and Hebei Chest Hospital. Each patient provided their informed consent before they were enrolled into NDIP.

Data Availability. The datasets generated during and/or analyzed during the current study are available from the corresponding author on reasonable request.

Open Access. This article is licensed under a Creative Commons Attribution-NonCommercial 4.0 International License, which permits any non-commercial use, sharing, adaptation, distribution and reproduction in any medium or format, as long as you give appropriate credit to the original author(s) and the source, provide a link to the Creative Commons licence, and indicate if changes were made. The images or other third party material in this article are included in the article's Creative Commons licence, unless indicated otherwise in a credit line to the material. If material is not included in the article's Creative Commons licence and your intended use is not permitted by statutory regulation or exceeds the permitted use, you will need to obtain permission directly from the copyright holder. To view a copy of this licence, visit http://creativecommons.org/licenses/by$\mathrm{nc} / 4.0 /$. 


\section{REFERENCES}

1. International Diabetes Foundation. Diabetes atlas. 9th ed. Brussels: IDF; 2019.

2. World Health Organization. Global tuberculosis report, 2019. WHO/CDS/TB/2019.20. Geneva: WHO, 2019.

3. Lutfiana NC, van Boven JFM, Masoom Zubair MA, Pena MJ, Alffenaar JC. Diabetes mellitus comorbidity in patients enrolled in tuberculosis drug efficacy trials around the world: a systematic review. Br J Clin Pharmacol. 2019;85(7):1407-17.

4. Huangfu P, Ugarte-Gil C, Golub J, Pearson F, Critchley J. The effects of diabetes on tuberculosis treatment outcomes: an updated systematic review and meta-analysis. Int $\mathrm{J}$ Tuberc Lung Dis. 2019;23(7):783-96.

5. Liu Q, Li W, Xue M, et al. Diabetes mellitus and the risk of multidrug resistant tuberculosis: a metaanalysis. Sci Rep. 2017;7(1):1090.

6. Kang YA, Kim SY, Jo KW, et al. Impact of diabetes on treatment outcomes and long-term survival in multidrug-resistant tuberculosis. Respiration. 2013;86(6):472-8.

7. Perez-Navarro LM, Restrepo BI, Fuentes-Dominguez FJ, et al. The effect size of type 2 diabetes mellitus on tuberculosis drug resistance and adverse treatment outcomes. Tuberculosis (Edinb). 2017;103: 83-91.

8. Munoz-Torrico M, Caminero Luna J, Migliori GB, et al. Comparison of bacteriological conversion and treatment outcomes among MDR-TB patients with and without diabetes in Mexico: preliminary data. Rev Port Pneumol (2006). 2017;23(1):27-30.

9. van Crevel R, Dockrell HM. TANDEM: understanding diabetes and tuberculosis. Lancet Diabetes Endocrinol. 2014;2(4):270-2.

10. WHO. The use of delamanid in the treatment of multidrug-resistant tuberculosis. Interim policy guidance. 2014. http://apps.who.int/iris/bitstream/ 10665/137334/1/WHO_HTM_TB_2014.23_eng.pdf. Accessed 16 Aug 2016.

11. Diacon AH, Pym A, Grobusch $M$, et al. The diarylquinoline TMC207 for multidrug-resistant tuberculosis. N Engl J Med. 2009;360(23):2397-405.

12. Diacon AH, Donald PR, Pym A, et al. Randomized pilot trial of eight weeks of bedaquiline (TMC207) treatment for multidrug-resistant tuberculosis: long-term outcome, tolerability, and effect on emergence of drug resistance. Antimicrob Agents Chemother. 2012;56(6):3271-6.

13. Schnippel K, Ndjeka N, Maartens G, et al. Effect of bedaquiline on mortality in South African patients with drug-resistant tuberculosis: a retrospective cohort study. Lancet Respir Med. 2018;6(9): 699-706.

14. DAIDS. Division of AIDS (DAIDS) table for grading the severity of adult and pediatric adverse events, version 2.1. 2017. https://rsc.niaid.nih.gov/sites/ default/files/daidsgradingcorrectedv21.pdf. Accessed 30 Sept 2020.

15. Dostalek M, Akhlaghi F, Puzanovova M. Effect of diabetes mellitus on pharmacokinetic and pharmacodynamic properties of drugs. Clin Pharmacokinet. 2012;51(8):481-99.

16. van Heeswijk RPG, Dannemann B, Hoetelmans RM. Bedaquiline: a review of human pharmacokinetics and drug-drug interactions. J Antimicrob Chemother. 2014;69(9):2310-8.

17. Hu M, Zheng C, Gao F. Use of bedaquiline and delamanid in diabetes patients: clinical and pharmacological considerations. Drug Des Devel Ther. 2016;10:3983-94.

18. Magee MJ, Kempker RR, Kipiani M, et al. Diabetes mellitus is associated with cavities, smear grade, and multidrug-resistant tuberculosis in Georgia. Int JTuberc Lung Dis. 2015;19(6):685-92.

19. Gao M, Gao J, Xie L, et al. Early outcome and safety of bedaquiline-containing regimens for treatment of MDR- and XDR-TB in China: a multicentre study. Clin Microbiol Infect. 2020. https://doi.org/10. 1016/j.cmi.2020.06.004.

20. Salhotra VS, Sachdeva KS, Kshirsagar N, et al. Effectiveness and safety of bedaquiline under conditional access program for treatment of drug-resistant tuberculosis in India: an interim analysis. Indian J Tuberc. 2020;67(1):29-37.

21. Borisov SE, Dheda K, Enwerem M, et al. Effectiveness and safety of bedaquiline-containing regimens in the treatment of MDR- and XDR-TB: a multicentre study. Eur Respir J. 2017;49(5):1700387.

22. Kim JH, Kwon OJ, Kim YS, Park MS, Hwang S, Shim TS. Bedaquiline in multidrug-resistant tuberculosis treatment: safety and efficacy in a Korean subpopulation. Respir Investig. 2020;58(1):45-51.

23. Mbuagbaw L, Guglielmetti L, Hewison C, et al. Outcomes of bedaquiline treatment in patients with multidrug-resistant tuberculosis. Emerg Infect Dis. 2019;25(5):936-43. 
24. Muñoz-Torrico M, Caminero-Luna J, Migliori GB, et al. Diabetes is associated with severe adverse events in multidrug-resistant tuberculosis. Arch Bronconeumol. 2017;53(5):245-50.

25. Borisov S, Danila E, Maryandyshev A, et al. Surveillance of adverse events in the treatment of drug-resistant tuberculosis: first global report. Eur Respir J. 2019;54(6):1901522.
26. Olayanju O, Esmail A, Limberis J, et al. A regimen containing bedaquiline and delamanid compared to bedaquiline in patients with drug-resistant tuberculosis. Eur Respir J. 2020;55(1):1901181.

27. Franke MF, Khan $\mathrm{P}$, Hewison $\mathrm{C}$, et al. Culture conversion in patients treated with Bedaquiline and/or Delamanid: a prospective multi-country study. Am J Respir Crit Care Med. 2021;203(1):111-19. 